Research Article

\title{
Deep-Learning-Based MRI Images for Analysis of Sport-Induced Ankle Joint Injury
}

\author{
Wenbo Zhang (iD) \\ Qiqihar University Sports Institute, Qiqihar 161006, Heilongjiang, China \\ Correspondence should be addressed to Wenbo Zhang; 01391@qqhru.edu.cn
}

Received 2 March 2021; Accepted 3 May 2021; Published 10 May 2021

Academic Editor: Gustavo Ramirez

Copyright ( $) 2021$ Wenbo Zhang. This is an open access article distributed under the Creative Commons Attribution License, which permits unrestricted use, distribution, and reproduction in any medium, provided the original work is properly cited.

\begin{abstract}
This study was to analyze the sport-induced ankle joint injury (AJI) images based on the neural network algorithms using the magnetic resonance imaging (MRI). 20 patients and 20 volunteers were included in the experimental and control groups, respectively. The hybrid diffusion equation (HDE) neural network (HDENN) algorithm was compared with the fully convolutional neural network (FCNN) and the FCNN preprocessing, and the HDE was applied to the MRI analysis of sport-induced AJI. The results showed that the total score of MRI image for the conventional position of the anterior talofibular ligament (ATFL) and posterior talofibular ligament (PTFL) was concentrated in $4(55 \%)$ and $5(65 \%)$, respectively. The number of patients with good prognosis with grade II injury (11 cases) was much higher than that of grade III injury (2 cases), and the number of patients with poor prognosis ( 4 cases) was lower than that of grade III injury ( 6 cases) $(P<0.05)$. Conventional MRI was recommended to observe the ATFL and PTFL, and the valgus position MRI was recommended for the calcaneofibular ligament (CFL); conservative treatment was recommended for patients with grades I and II AJI, but surgical treatment was recommended for patients with grade III AJI.
\end{abstract}

\section{Introduction}

Located between the lower leg and the foot of the human body, the ankle joint mainly undertakes the weight-bearing function of the human body during exercise. It mainly relies on the cooperation of ligaments and tendons to maintain its stability [1]. AJI is a common bone injury in life, which refers to the ankle joint swelling and pain due to excessive stretching and tearing of the ankle joint ligament [2]. In clinical medicine, the most common AJIs include joint ligament injury, dislocation, and fracture [3]. Most AJIs are caused by fixed and rapid foot movement, sudden inversion, and adduction of the ankle joint, causing ligament tears and injuries and severe cases with ankle fractures [4]. If the treatment is not performed in time, or the treatment is not thorough, it will cause repeated sprains in the future and seriously affect the normal function of the joints. Therefore, it is necessary for clinical medical staff to master anatomical knowledge of ankle joints, find the ankle joint and surrounding ligament injuries in time, and adopt corresponding treatment measures.
In recent years, AJI can be inspected with clinical pathology, computer tomography (CT), ultrasound, and MRI [5]. When the ankle ligament is acutely injured, the patient is unable to cooperate due to pain and swelling of the ankle join, which increases the difficulties of clinical pathological examination. In recent years, with the development of bioimaging, CT examination plays a very important role in fracture classification and to a certain extent provides imaging support for clinical treatment. However, due to its lack of clarity and resolution, the final image cannot completely show the ligament injury and the condition of the parenchyma, making it unable to be used in the inspection of the ankle ligament [6]. MRI is a medical imaging technique used in radiology. It uses the combined action of strong magnetic fields, magnetic field gradients, and silent electric waves to form images of human organs, so as to analyze physiological processes or anatomical images of the body [7]. MRI imaging is widely used in the skeletal system. Liu et al. [8] used MRI imaging to diagnose the AJI and found that MRI imaging is highly sensitive in diagnosing ligament injuries. 
Blissett et al. [9] combined the conventional MRI images and used various postural MRI to study AJI, providing a good reference for clinical diagnosis of ankle joints. The importance of computer processing images in clinical applications cannot be ignored. The mathematical algorithms used play an inestimable role. However, the current MRI imaging based on neural network algorithms is not mature enough. In this study, the mixed diffusion equation could process objective phenomena into MRI images and perform special processing on the images [10].

The HDE neural network algorithm proposed in this study was compared with FCNN and FCNN preprocessing for effective comparison and analysis, and it was applied to the MRI image analysis of sport-induced AJI. The objective of studying AJI and pathological grading was to detect whether MRI images can effectively assess motor AJI and provide a simple and efficient diagnosis method for the clinic.

\section{Materials and Methods}

2.1. Research Subjects. 20 sport-induced AJI patients who were treated in the hospital from November 1, 2019, to September 28, 2020, were selected and included in the experimental group, and 20 healthy volunteers were selected and included in the control group. There were 23 males and 17 females, with the average age of $49.58 \pm 12.54$ years. The experiment had been approved by the Medical Ethics Committee of Hospital, and the patients and their families had understood the research situation and signed the informed consent forms.

The inclusion criteria were defined as follows: patients who were clinically diagnosed as AJI, patients with complete clinical diagnosis data, and patients with good quality of MRI image.

The exclusion criteria were defined as follows: patients who had undergone the ankle joint anatomy surgery; patients who suffered from ankle fractures caused by violence; patients who suffered from arthritis, osteoporosis, and joint infections; and patients with contraindication of MRI scans that were able to cooperate the MRI scans.

2.2. MRI Technology. The research subjects were scanned with a 3.0T magnetic resonance instrument manufactured by Magnetom Trio Tim System, Siemens. During the scan, the patients and volunteers were asked to be in a supine position, with the knee joints straightened and relaxed. When the conventional ankle joint plantar was bent for around $25^{\circ}$ in valgus position, the scanning range was determined as follows: it was scanned from up to down, starting from the inferior tibiofibular syndesmosis at the top and ending at the lower edge of the root bone at the bottom. All MRI images in this study were completed by experienced radiologists. T1WI and T2WI were selected to scan the cross-sectional, sagittal, coronal, and valgus position of the ankle joint, respectively. Scanning parameters were as follows: the matrix was $251 \times 251$, the layer thickness was $3.5 \mathrm{~mm}$, the field of view was $25 \times 25 \mathrm{~cm}$, the flip angle was $15^{\circ}$, and the layer spacing was $6.1 \mathrm{~mm}$.
2.3. The Scoring Standard and Grades of MRI Images of Ankle Ligament Injury. The scoring standard of ankle ligament MRI images was as follows: 0 point indicates that the basic structure of the ligaments cannot be identified; 1 point indicates that only a small part of the ligament can be observed, which was dotted or patchy; 2 points indicate that most of the ligaments were visible, and the beginning or end of the ligament can be clearly seen, showing a strip-shaped cross-sectional signal; 3 points indicate that the beginning and end of the ligament can be observed, clearly showing the full length of the ligament. The pathological classification of MRI images of ankle ligament injury is as follows: grade I suggests that the basic structure of the ligament was complete but with swelling; grade II suggests that the ligament was in irregular shape and the fiber bundles were torn, showing high signal; and grade III suggests that the ligament was broken and discontinuous, and the whole paragraph was in wave shape.

2.4. Analysis Process of MRI Image for Ankle Joint. All ankle joint images were completed and discussed by two senior imaging diagnosticians. The ankle ligaments in different positions were observed to analyze the MRI images, so as to evaluate the specific situation of the ankle ligament injury under the normal position MRI and valgus position MRI, respectively. The normal position MRI images were compared with valgus position MRI examinations to explain the changes in the ankle ligaments and to classify the ligament injuries. The ankle images of 20 volunteers were scored for diagnosis, and then the coronal and sagittal scores of each image were added together, and the final score was undertaken as the standard for evaluating the final effect. $20 \mathrm{AJI}$ patients received conservative treatment, and the results of normal position and valgus position MRI were compared during the treatment.

2.5. Neural Network Algorithm Based on the HDE. The basic unit of the neural network model was the neuron (as shown in Figure 1), which was a general neuron model with $m$ data inputs. The vector $a=\left(a_{1}, a_{2}, \ldots, a_{m}\right)^{T}$ was the input of the neuron and $\omega=\left(\Phi_{1}, \varpi_{2}, \ldots, \varpi_{m}\right)^{T}$ was an adjustable input weight. $\beta$ represented the offset signal and was the threshold of the neuron. $v$ referred to the basis function of neuron and was a multiple data input, the single data output function was represented with $v(a, \omega, \beta)$, and $g(v)$ represented the activation function, which acted on a nonlinear mapping and processed the data output of the basis function.

The algorithm proposed in this study was converted so as to satisfy the following nonlinear diffusion equation:

$$
\frac{\partial v}{\partial s}=\delta \operatorname{div}\left(|\Delta v|^{k-2} \nabla v\right)+(1-\delta) \operatorname{div}\left(|\nabla v|^{l-2} \nabla v\right)-\zeta(v-g),
$$

$\left.v\right|_{t=0}=g$, 


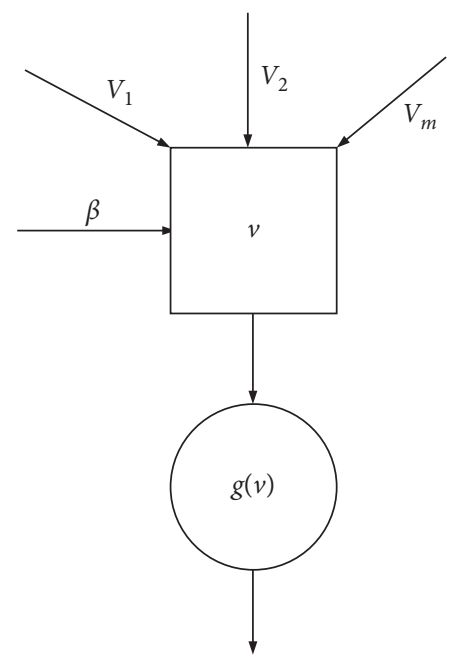

FIGURE 1: Schematic diagram of neuron.

$$
\left.\frac{\partial v}{\partial m}\right|_{\partial \Omega}=0
$$

In equations (1)-(3), $g$ represented the initial noise image; $v$ represented the basis function of the neuron, which was multiple data inputs; $a \in \Omega$ represented the two-dimensional area range of the image, which satisfied $k>1,0<l<1,0<\delta<1$. For the gradient term, the forward or backward difference method was adopted for numerical discretization:

$$
\begin{aligned}
& C_{a}^{ \pm} v_{i, j}=\frac{ \pm\left[v_{i \pm 1, j}-v_{i, j}\right]}{t}, \\
& C_{b}^{ \pm} v_{i, j}=\frac{ \pm\left[v_{i, j \pm 1}-v_{i, j}\right]}{t} .
\end{aligned}
$$

Equations (4) and (5) were discretized continually. The vector $a=\left(a_{1}, a_{2}, \ldots, a_{m}\right)^{T}$ represented the input vector of the neuron and $v$ referred to the basis function of the neuron, which could be calculated with the following equations:

$$
\begin{aligned}
& P_{1 i, j}^{m}=\frac{C_{a}^{ \pm} v_{i, j}^{m}}{\left(\left(C_{a}^{ \pm} v_{i, j}^{m}\right)^{2}+\left(C_{b}^{ \pm} v_{i, j}^{m}\right)^{2}\right)^{(2-k) / 2}}, \\
& P_{2 i, j}^{m}=\frac{C_{b}^{ \pm} v_{i, j}^{m}}{\left(\left(C_{a}^{ \pm} v_{i, j}^{m}\right)^{2}+\left(C_{b}^{ \pm} v_{i, j}^{m}\right)^{2}\right)^{(2-k) / 2}} .
\end{aligned}
$$

In equations (6) and (7) above, $P=|\nabla v|^{k-2} \nabla v$ and $Q=$ $|\nabla v|^{l-2} \nabla v$ could be satisfied, and $v$ represented the basis function of the neuron. To ensure the stability of the data in the equation, when the forward difference was selected, the external difference format should be backward, and the following equation could be obtained:

$$
\operatorname{div}(P)_{i, j}^{m}=C_{a}^{-}\left(P_{1 i, j}^{m}\right)+C_{b}^{-}\left(P_{2 i, j}^{m}\right) .
$$

In equation (8), $0 \leq i \leq I, 0 \leq j \leq J$ was satisfied. $I$ represented the number of pixels in the horizontal direction of the image; $J$ represented the number of pixels in the vertical direction of the image; $P=|\nabla v|^{k-2} \nabla v$; and $v$ referred to the basis function of the neuron. Equation (8) was continued to be discretized, and then the following equations could be obtained:

$$
\begin{aligned}
Q_{1 i, j}^{m} & =\frac{C_{a}^{ \pm} v_{i, j}^{m}}{\left(\left(C_{a}^{ \pm} v_{i, j}^{m}\right)^{2}+\left(C_{b}^{ \pm} v_{i, j}^{m}\right)^{2}\right)^{(2-l) / 2}}, \\
Q_{2 i, j}^{m} & =\frac{C_{b}^{ \pm} v_{i, j}^{m}}{\left(\left(C_{a}^{ \pm} v_{i, j}^{m}\right)^{2}+\left(C_{b}^{ \pm} v_{i, j}^{m}\right)^{2}\right)^{(2-l) / 2}} \\
\operatorname{div}(Q)_{i, j}^{m} & =C_{a}^{-}\left(Q_{1 i, j}^{m}\right)+C_{b}^{-}\left(Q_{2 i, j}^{m}\right) .
\end{aligned}
$$

In equations (9)-(11), $0 \leq i \leq I, 0 \leq j \leq J$ was satisfied. $I$ represented the number of pixels in the horizontal direction of the image; $J$ represented the number of pixels in the vertical direction of the image; and $v$ referred to the basis function of the neuron. Then, the equation was discretized under the external discrete phase, using a combination of forward and backward difference methods, the equations of which could be given as follows:

$$
\begin{aligned}
v_{i, j}^{m+1}= & v_{i, j}^{m}+\sigma\left(\beta \operatorname{div}(P)_{i, j}^{m}\right) \\
& +(1-\beta) \operatorname{div}(Q)_{i, j}^{m}-\sigma \zeta^{m}\left(v_{i, j}^{m}-g_{i, j}\right), \\
\zeta^{m}= & \sum_{i, j}\left(\left(\beta \operatorname{div}(P)_{i, j}^{m}\right)\right. \\
& \left.+(1-\beta) \operatorname{div}(Q)_{i, j}^{m}\left(v_{i, j}^{m}-g_{i, j}\right)\right)\left(\varphi^{2} I J\right)^{-1} .
\end{aligned}
$$

In equations (12) and (13), $\beta$ represented the offset signal, which was the threshold of the neuron. $P=|\nabla v|^{k-2} \nabla v$ and $0 \leq i \leq I, 0 \leq j \leq J$ were satisfied. $I$ represented the number of pixels in the horizontal direction of the image; $J$ represented the number of pixels in the vertical direction of the image; and $v$ referred to the basis function of the neuron. When the initial boundary value was discretized, the following conditions should be met:

$$
\begin{aligned}
& v_{i, j}^{0}=g_{i, j}=g(i t, j t), \\
& v_{i, 0}^{m}=v_{i, 1}^{m}, v_{0, j}^{m}=v_{1, j}^{m} .
\end{aligned}
$$

In equations (14) and (15), I represented the number of pixels in the horizontal direction of the image; $J$ represented the number of pixels in the vertical direction of the image; $v$ referred to the basis function of the neuron; and $g$ represented the initial noise image.

2.6. Statistical Methods. The IBM SPSS Statistics 20.0 software was adopted for statistical analysis of data. The data conforming to the normal distribution was expressed by the mean \pm variance $(\bar{x} \pm s)$, and the MRI measurement values of the three groups of ligaments were compared; if the 
calculated data failed to meet the normal distribution, it was expressed by frequency. The examinations of normal position and valgus position MRI and the surgery determination were tested with the Kappa test. $P<0.05$ indicated that the difference was statistically significant.

\section{Results}

3.1. Comparison on Segmentation Performance of Three Algorithms. The results in Figure 2 illustrated that the performance of the HDENN algorithm was not greatly improved compared with FCNN and FCNN preprocessing in the complete area, and the evaluation index was only improved by 0.01 . In the core area, the HDENN was increased by 0.13 compared to FCNN; but the difference was not obvious compared with the FCNN preprocessing. In the enhancement area, the HDENN algorithm showed an obvious improvement over the FCNN algorithm, and the evaluation index was increased by 0.17 . The HDENN algorithm showed nonideal evaluation index in the complete area but showed good segmentation performance in the core area and the enhanced area, with prominent evaluation index. Therefore, the HDENN algorithm in this study had better segmentation performance.

\subsection{Comparison on Basic Data of Patients in Two Groups.} There were 11 males (55\%) and 9 females (45\%) in the experimental group and 12 males (60\%) and 8 females (40\%) in the control group. There was no visible difference in gender for patients between the two groups $(P>0.05)$ (as given in Figure 3 ). There was no great difference in age between the two groups of patients $(P>0.05)$ (as illustrated in Figure 4).

3.3. Images of AJI with MRI Examination. Figure 5 shows an image of ATFL with partial break in grade II, and Figure 6 shows an image of CFL with complete break in grade III. Both sagittal and coronal T1WI and T2WI displayed the full length of the ATFL, CFL, and PTFL. Among them, the sagittal view showed a low signal and oval shape. In MRI images, the overall orientation of the three ligaments could be observed, and the tendon sheaths around the ligaments could be accurately observed.

It was a quicker way to find ATFL on the level where PTFL appeared. Because the PTFL was relatively wide and it was not easy to damage and break, ATFL started from the anterior edge of the lateral malleolus and then extended medially and ended at the bone neck. ATFL appeared at the level of the talus neck. Compared with the wide fan shape of PTFL, ATFL was relatively long and slender. In tomographic MRI, ATFL could be found in 3 4 levels along the lateral malleolus tip to the proximal end. The width of the ATFL was about 7 11 mm, and the thickness of the MRI layer was $3 \mathrm{~mm}$. If it could not be found due to no scanning, it was suspected to be broken. Because ATFL was $6 \sim 10 \mathrm{~mm}$ in thickness, it may be seen on two levels. ATFL and PTFL were basically at right angles to the long axis of the tibia and fibula. When the ankle was scanned in the neutral position, the full

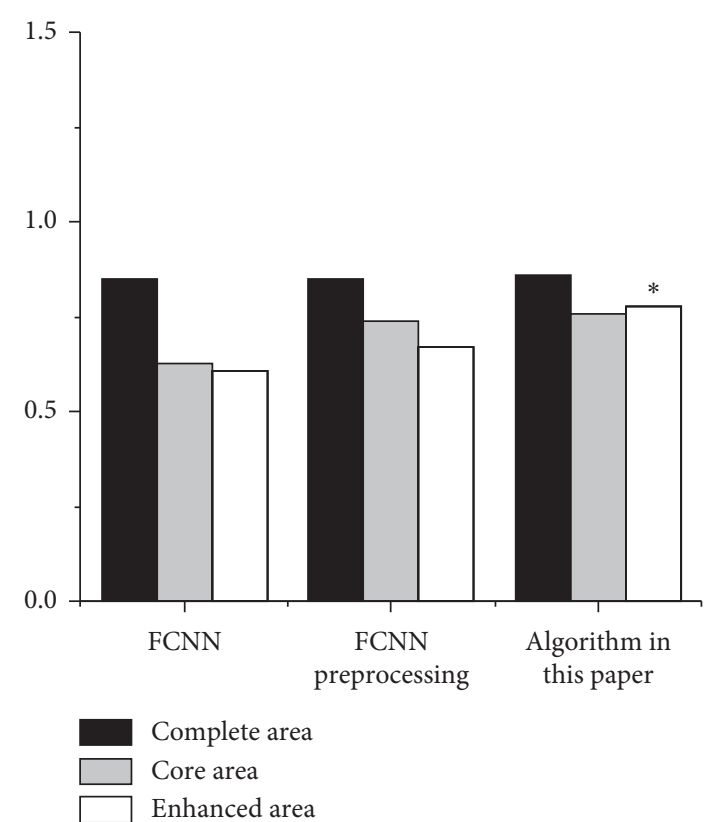

Figure 2: Comparison on segmentation performances of three algorithms. Note: ${ }^{*}$ indicated observable difference in contrast to FCNN $(P<0.05)$.

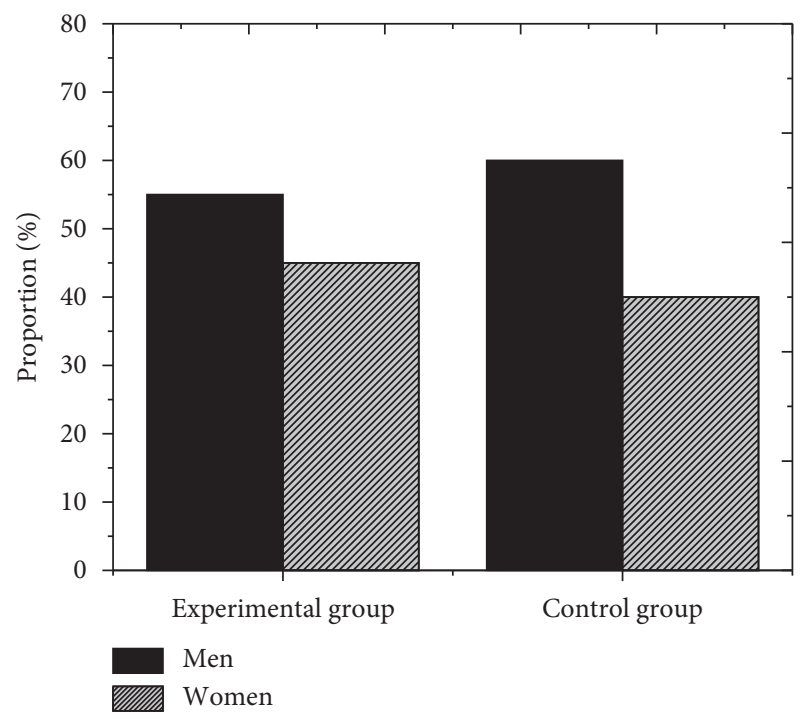

Figure 3: Comparison on genders of patients in two groups.

length of ATFL and PTFL could be seen on the horizontal plane. CFL and the distal end of the tibial long axis were clamped $16-22^{\circ}$ backwards, which indicated that the ankle joint was scanned when the back extension was $20^{\circ}$, and the full length of the CFL could be seen in the sagittal and coronal planes. When the ankle joint was scanned in the neutral position, part of the CFL could be found on the horizontal plane.

3.4. Comparison of Three Ligaments in MRI Images. The ankle MRI images of 20 volunteers were scored, and the total scores of ATFL, CFL, and PTFL were calculated accordingly. 


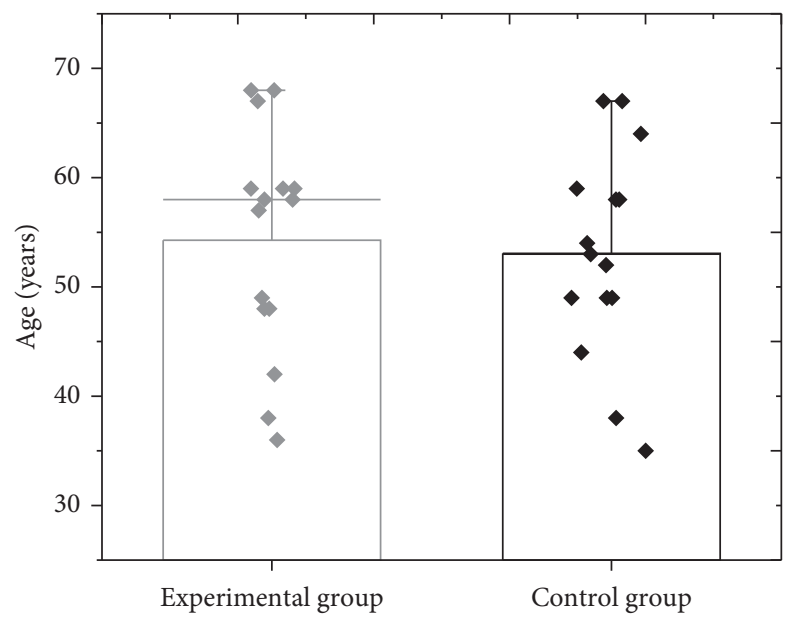

FIgURE 4: Age distribution of patients in two groups.

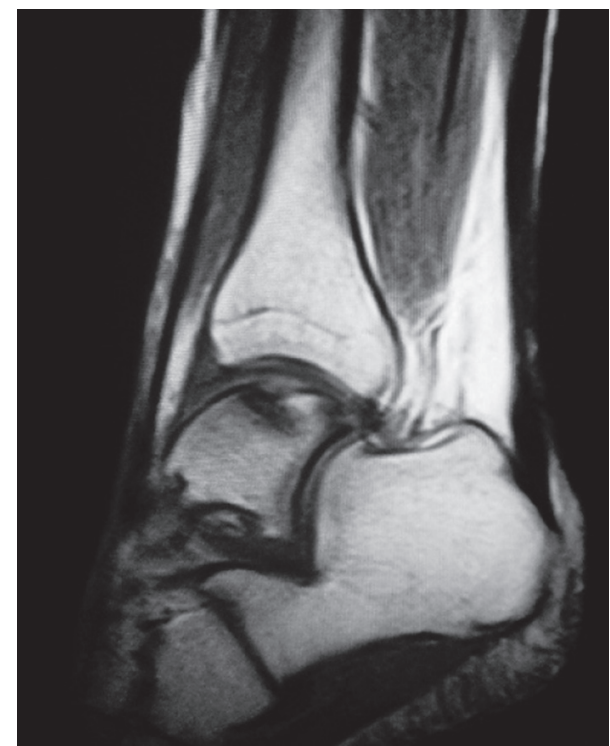

Figure 5: Partial break of anterior talofibular ligament (grade II).

Figure 7 shows that the total score of ATFL in both normal position and valgus position was concentrated in 4 points, accounting for $55 \%$ and $45 \%$, respectively; and the total score of plantar flexion position was concentrated in 3 points. The total score of CFL in normal position and valgus position was concentrated in 2 points and 4 points, respectively; and the total score of plantar flexion position was concentrated on 3 points. The total score of PTFL in both the normal and valgus positions was concentrated on 5 points, accounting for $65 \%$ and $60 \%$, respectively (as shown in Figure 8), and the total score of plantar flexion was concentrated in 6 points.

3.5. Comparison on Measurement Values of Three Ligaments in MRI. The ankle ligaments indexes of 20 cases measured by the MRI image were inputted in the IBM SPSS Statistics 20.0 software for analysis. It was found that there was no statistical

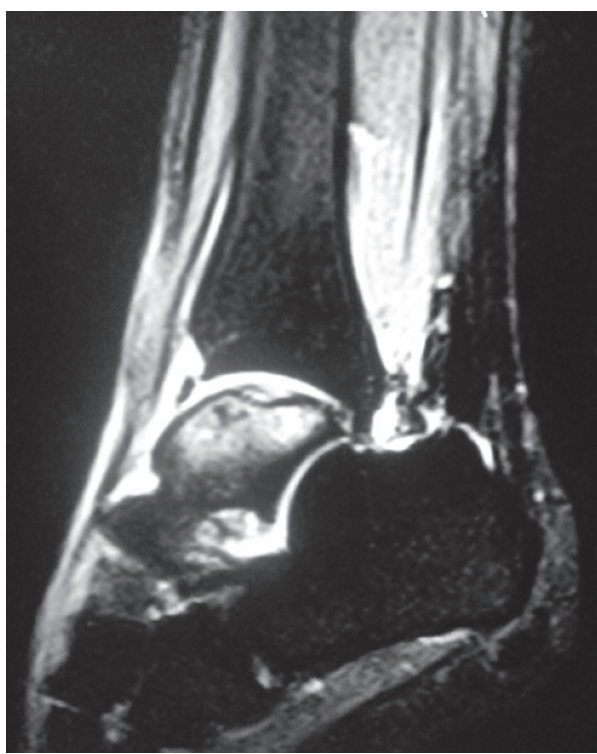

FIGURE 6: Complete break of calcaneofibular ligament (grade III).

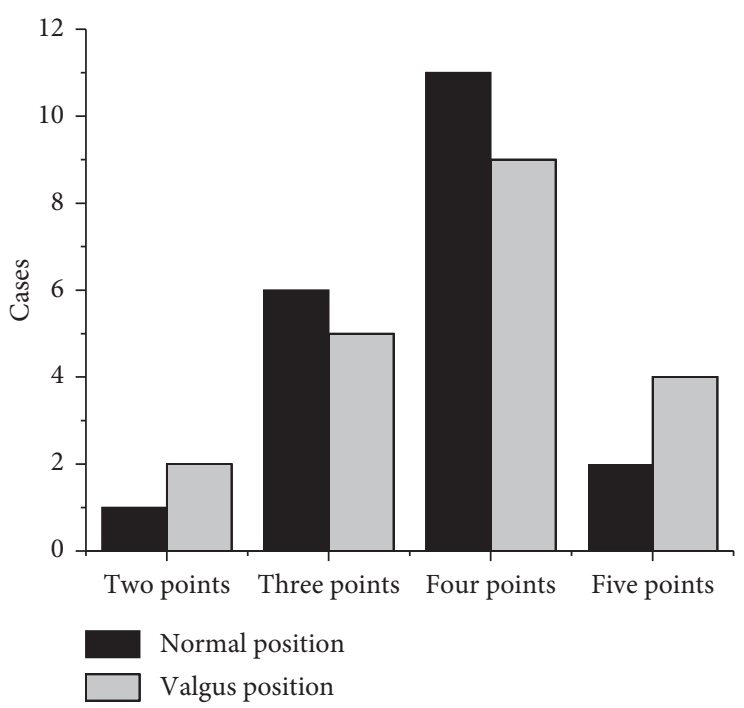

Figure 7: Scores of anterior talofibular ligament.

difference in the average length $(14.645 \pm 0.576 \mathrm{~mm})$, width $(8.552 \pm 0.832 \mathrm{~mm})$, and thickness $(1.845 \pm 0.157 \mathrm{~mm})$ of ATFL (as illustrated in Figure 9$)(P>0.05)$. There was no statistical difference in the average length $(23.565 \pm 1.567 \mathrm{~mm})$, width $(5.735 \pm 0.456 \mathrm{~mm})$, and thickness $(2.167 \pm 0.256 \mathrm{~mm})$ of CFL (as illustrated in Figure 10$)(P>0.05)$. In addition, there was no statistical difference in the average length $(19.656 \pm 1.462 \mathrm{~mm})$, width $(6.456 \pm 0.257 \mathrm{~mm})$, and thickness $(2.576 \pm 0.256 \mathrm{~mm})$ of PTFL (as illustrated in Figure 11) $(P>0.05)$.

3.6. Comparison on Grades Determined by Normal Position MRI, Valgus Position MRI, and Surgery Treatment. The 20 AJI patients had 56 ligaments in total, including 21 in grade II and 19 in grade III. The patients were tested at the normal and valgus position MRI images, and the results were 


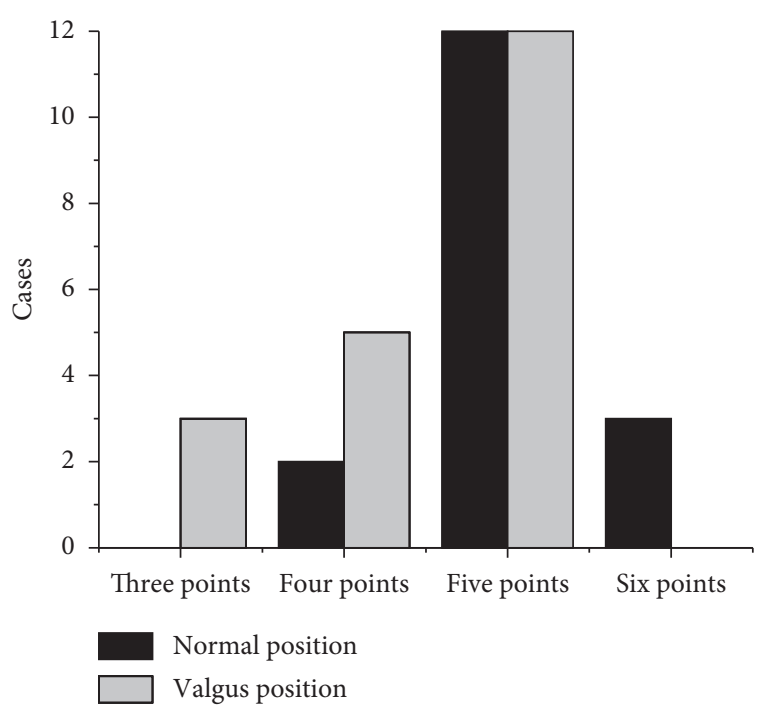

FIGURE 8: Scores of posterior talofibular ligament.

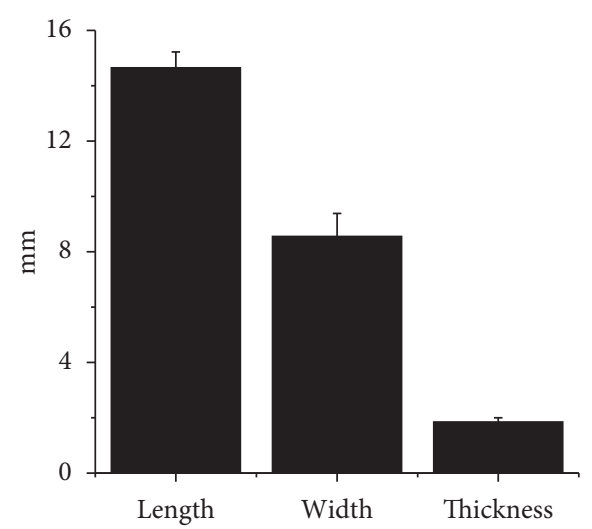

FIGURE 9: MRI measurement values of anterior talofibular ligament.

counted and graded. The grade II injury in ATFL patients detected by the normal position MRI ( 9 cases) and the surgical diagnosis (12 cases) showed considerable difference from each other (Kappa $=0.789, P<0.05)$; the grade III ATFL detected with the valgus position MRI (8 cases) was observably different from that detected with the surgical diagnosis ( 13 cases) (Kappa $=0.834, P<0.05)$ (as disclosed in Figure 12). Figure 13 illustrates that grade II of CFL detected by normal position MRI (11 cases) was greatly different from that by the surgical diagnosis (19 cases) (Kappa $=0.856$, $P<0.05$ ); grade III of CFL detected by the valgus position MRI and surgical diagnosis were visibly different (Kappa $=0.956, P<0.05)$. There were 2 cases in grade II PTFL and 0 cases in grade III detected by the normal position MRI, valgus position MRI, and surgical diagnosis.

\subsection{Comparison on Conservative Treatment Effects of} Ligament. 20 patients were treated conservatively, and the patients were followed up for about 10 months on average. During the plaster-assisted recovery period, the patient was instructed to properly perform joint recovery training and

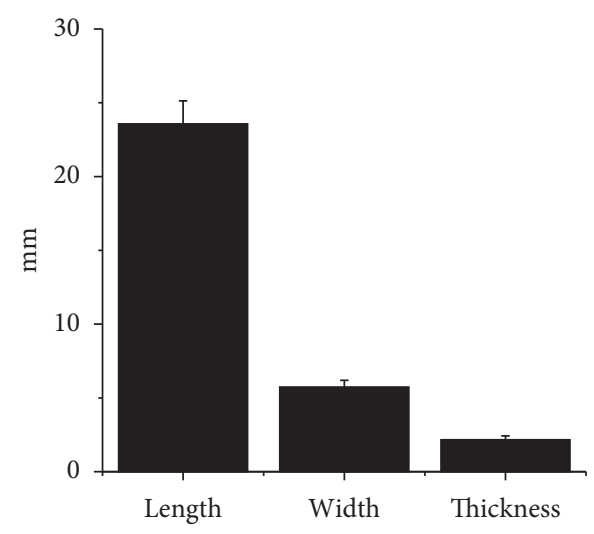

FIgURE 10: MRI measurement values of calcaneofibular ligament.

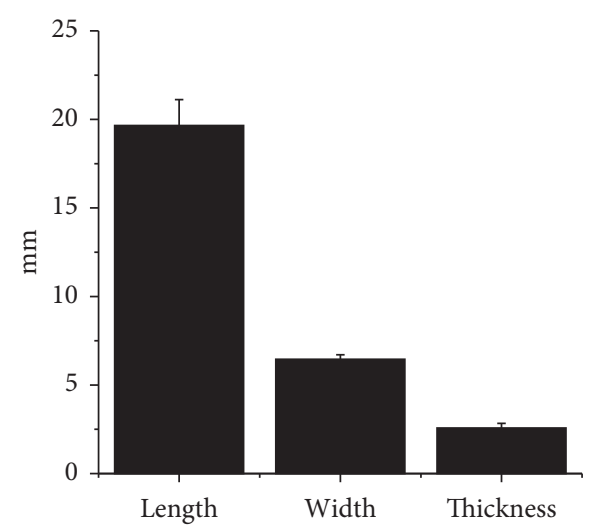

FIGURE 11: MRI measurement values of posterior talofibular ligament.

not to go to the ground too early. During the recovery period, there was no ankle swelling, no pain, no difference in walking with normal people, and no more ankle sprains during the follow-up process. The AJI status during the follow-up was recorded and analyzed statistically. After conservative treatment of 20 patients with ankle ligament injury, there was no statistically huge difference between grade I and grade II injuries $(P>0.05)$; the difference between the treatment results of grade I injury and grade III injury was extremely great $(P<0.05)$. The prognosis of patients with grade II injury (11 cases) was much higher than that of grade III injury (2 cases), and the prognosis (4 cases) was lower than that of grade III injury (6 cases), showing statistically obvious difference $(P<0.05)$ (as revealed in Figure 14). The analysis of conservative treatment results indicated that the grade I and grade II injuries could achieve better results in contrast to the grade III injuries.

\section{Discussion}

In medicine, the ankle joint is also called the ankle calf joint. When people walk and exercise in life, the weight of the body is concentrated on this part. Therefore, this part is the largest weight-bearing joint in the human body, which completes the necessary movements of human body through the 


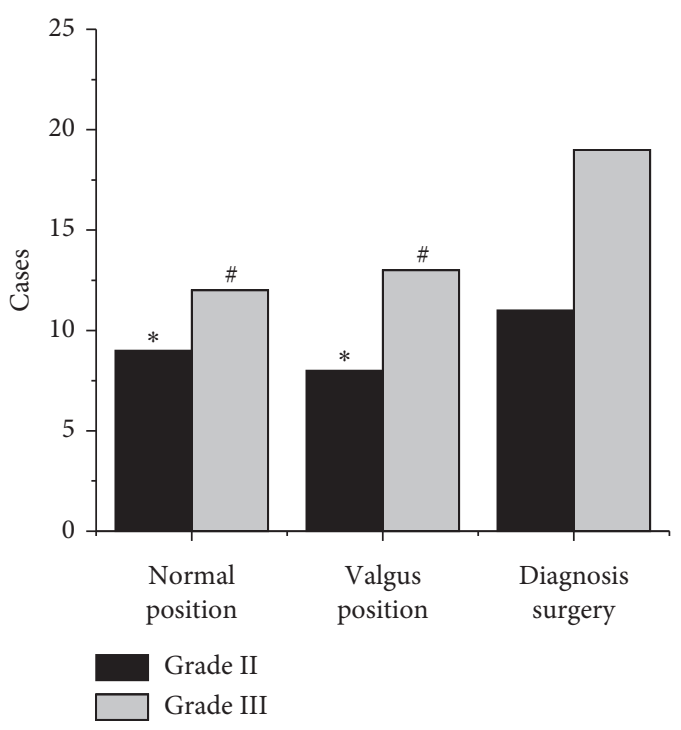

FIGURE 12: Grades of anterior talofibular ligament detected by three ways. Note: *indicates observable difference in contrast to the grade II injury detected by surgical diagnosis $(P<0.05)$; \#suggests remarkable difference in contrast to the grade III injury detected by surgical diagnosis $(P<0.05)$.

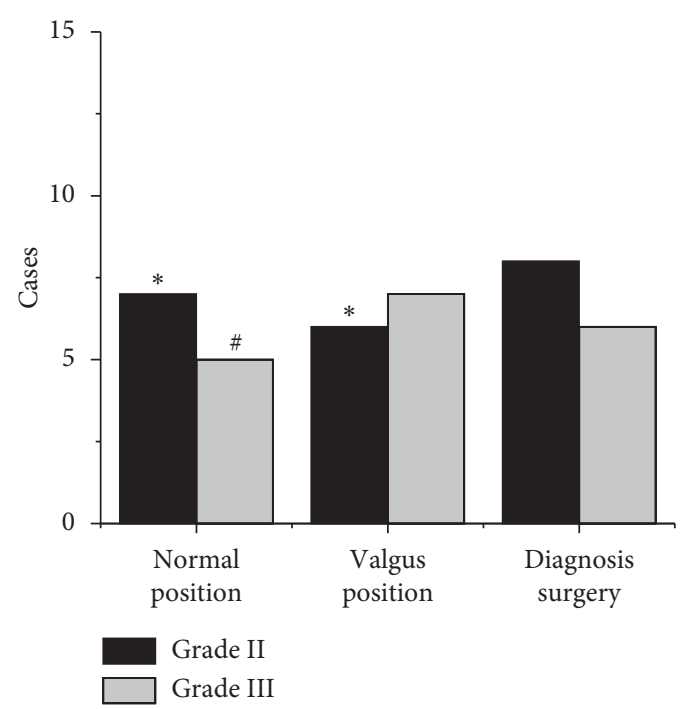

FIGURE 13: Grades of calcaneofibular ligament detected by three ways. Note: ${ }^{*}$ indicates observable difference in contrast to the grade II injury detected by surgical diagnosis $(P<0.05)$; \#suggests remarkable difference in contrast to the grade III injury detected by surgical diagnosis $(P<0.05)$.

extension, plantar flexion, and rotation of the ankle joint [11]. The ankle joint is mainly composed of three parts, the tibia, medial fibula, and lateral talus, which are important pulley joints of the human body. With the continuous development of society, more attention has been paid to medical treatment, and people have a deeper understanding of AJI. Nowadays, clinical pathology, CT, and ultrasound are not enough to fully support a full set of examinations of the ankle joint [12]. The study uses high-potential MRI images to

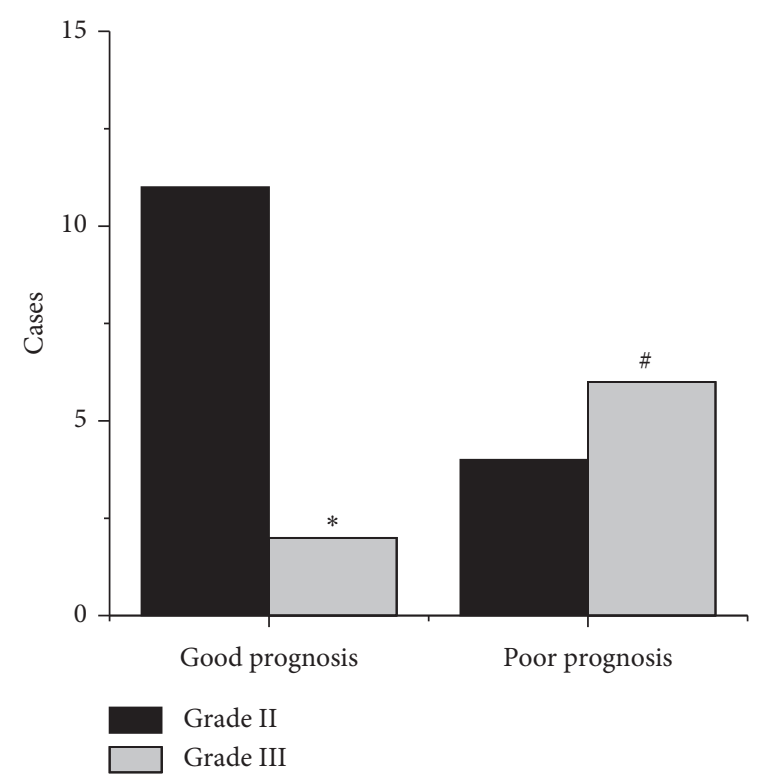

FIgure 14: Comparison on conservative treatment effects of ligament. Note: * suggests observable difference in contrast to good prognosis of the grade II injury $(P<0.05)$; \#indicates great difference in contrast to poor prognosis of the grade II injury $(P<0.05)$.

assess the sport-induced AJI, which can effectively make up for the lack of clinical diagnosis at this stage.

Yamauchi et al. [13] took 35 AJI patients as the research subjects for MRI imaging examination of the external ankle ligaments and found that the cross-sectional image was the clearest. The MRI images of the valgus position in this study were also clearer and showed better results. You et al. [14] used multiposition MRI ankle ligament scans and found that, at the injury site, the ligaments were passively elongated with swelling and thickening; the ligaments were not continuous but discontinuous; and some parts were shortened, so that the image presented a mixed signal of high and low, which was consistent with the results of this study. During the scanning process of MRI for lateral ankle ligament injuries, MRI images show different sensitivities for different ligaments, which may increase the clinical missed diagnosis rate and misdiagnosis rate [15]. Clanton et al. [16] adopted different magnetic field strengths and various joint coils to improve the MRI images. Studies have shown that low magnetic field strengths can better assess the ankle ligament injuries. In this study, low magnetic field strength MRI was employed to evaluate the AJI patients. It was found that the images of ATFL and PTFL in the sagittal position were clearer, which was consistent with above study. It was found that there was no statistical difference in the average length $(14.645 \pm 0.576 \mathrm{~mm})$, width $(8.552 \pm 0.832 \mathrm{~mm})$, and thickness $(1.845 \pm 0.157 \mathrm{~mm})$ of ATFL $(P>0.05)$. There was no statistical difference in the average length $(23.565 \pm 1.567 \mathrm{~mm})$, width $(5.735 \pm 0.456 \mathrm{~mm})$, and thickness $(2.167 \pm 0.256 \mathrm{~mm})$ of CFL $(P>0.05)$. In addition, there was no statistical difference in the average length $(19.656 \pm 1.462 \mathrm{~mm})$, width $(6.456 \pm 0.257 \mathrm{~mm})$, and thickness $(2.576 \pm 0.256 \mathrm{~mm})$ of PTFL $(P>0.05)$. 
Scientific Programming

If there is any AJI, the action should be stopped immediately. If possible, ice compress should be applied immediately. If it is bleeding, it should be bandaged to reduce the amount of bleeding. In clinical practice, protective gear, plaster, and wide tape are often applied for fixation [17]. Based on the physical examination of the patient, the injury is graded and evaluated, and the next treatment plan is reasonably arranged according to the actual injury condition. Under normal circumstances, conservative treatment can be performed when the ankle ligament is partially ruptured. If it is completely ruptured or fractured, surgical treatment is required [18]. Although there is a lot of relevant literature on AJI, a standard treatment that is widely supported by medicine has not yet appeared. In this study, 20 AJI patients were treated conservatively and followed up. The statistical analysis revealed that there was no extremely huge difference between ligament grade I and grade II injuries $(P>0.05)$; ligament grade I and grade III injuries were much different from each other $(P<0.05)$. The prognosis of patients with grade II injury (11 cases) was much higher than that of grade III injury (2 cases), and the prognosis (4 cases) was lower than that of grade III injury (6 cases), showing statistical obvious difference $(P<0.05)$. According to the analysis of conservative treatment results, the effects of conservative treatment for grade I and II injuries were more obvious than those for grade III injury.

\section{Conclusion}

The HDE neural network algorithm proposed in this study was compared with FCNN and FCNN preprocessing for effect comparison analysis, and the HDE was applied to the MRI image analysis of sport-induced AJI. It was found that the evaluation index in the complete area was not very ideal, but the segmentation performance of the algorithm in the core area and the enhanced area was very good. The normal position MRI images showed better observation effects on ATFL and PTFL, and valgus position MRI showed better observation effects on the three ligaments; it was an ideal position for patients to perform MRI scans in a natural state; the AJI patients in grade I and grade II could be treated conservatively, but surgical treatment was recommended for patients with grade III injuries. The shortcomings of this study were the shorter time to collect clinical data, fewer medical records of patients, and the high cost of MRI examinations. Due to economic constraints, some patients withdrew from the study due to incomplete imaging data. Later, it will consider expanding the sample range, so as to provide appropriate subsidies in economic aspects to enhance the scientific nature of research. In short, MRI was adopted to evaluate the AJI, which was conductive to further understand the MRI imaging characteristics of the ankle joint, helping the clinician be familiar with the specific morphology of the ankle joint ligaments, performing timely diagnosis and treatment, and improving the prognosis.

\section{Data Availability}

No data were used to support this study.

\section{Conflicts of Interest}

The authors declare that they have no conflicts of interest.

\section{Acknowledgments}

This work was supported by the project of Philosophy and Social Sciences Research in Heilongjiang Province in 2018 (no. 18TYB097), Qiqihar City in Heilongjiang Province Science and Technology Bureau 2019 Achievements of Science and Technology Plan General Projects (GYGG201912), and College Students' Innovative Entrepreneurial Training Plan in 2020 in Heilongjiang Province General Project (202010232129).

\section{References}

[1] M. K. Kim and Y. J. Shin, "Immediate effects of ankle balance taping with kinesiology tape for amateur soccer players with lateral ankle sprain: a randomized cross-over design," Medical Science Monitor, vol. 23, pp. 5534-5541, 2017.

[2] E. Delahunt, C. M. Bleakley, D. S. Bossard et al., "Clinical assessment of acute lateral ankle sprain injuries (ROAST): 2019 consensus statement and recommendations of the international ankle consortium," British Journal of Sports Medicine, vol. 52, no. 20, pp. 1304-1310, 2018.

[3] S. Yamaguchi, R. Akagi, S. Kimura et al., "Avulsion fracture of the distal fibula is associated with recurrent sprain after ankle sprain in children," Knee Surgery, Sports Traumatology, Arthroscopy, vol. 27, no. 9, pp. 2774-2780, 2019.

[4] C. M. Bleakley, J. B. Taylor, S. L. Dischiavi, C. Doherty, and E. Delahunt, "Rehabilitation exercises reduce reinjury post ankle sprain, but the content and parameters of an optimal exercise program have yet to be established: a systematic review and meta-analysis," Archives of Physical Medicine and Rehabilitation, vol. 100, no. 7, pp. 1367-1375, 2019.

[5] G. M. LiMarzi, K. F. Scherer, M. L. Richardson et al., "CT and MR imaging of the postoperative ankle and foot," Radiographics, vol. 36, no. 6, pp. 1828-1848, 2016.

[6] C. Gao, Z. Chen, Y. Cheng et al., "Comparative anatomy of the mouse and human ankle joint using Micro-CT: utility of a mouse model to study human ankle sprains," Mathematical Biosciences and Engineering, vol. 16, no. 4, pp. 2959-2972, 2019.

[7] C. L. Fisher, T. Rabbani, K. Johnson et al., "Diagnostic capability of dynamic ultrasound evaluation of supination-external rotation ankle injuries: a cadaveric study," $B M C$ Musculoskeletal Disorders, vol. 20, no. 1, p. 502, 2019.

[8] W. Liu, H. Li, and Y. Hua, "Quantitative magnetic resonance imaging (MRI) analysis of anterior talofibular ligament in lateral chronic ankle instability ankles pre- and postoperatively," BMC Musculoskeletal Disorders, vol. 18, no. 1, p. 397, 2017.

[9] S. Blissett, M. Chetrit, B. Kovacina, V. Mardigyan, and J. Afilalo, "Performing cardiac magnetic resonance imaging in patients with cardiac implantable electronic devices: a contemporary review," Canadian Journal of Cardiology, vol. 34, no. 12, pp. 1682-1686, 2018.

[10] A. Panda, G. O’Connor, W. C. Lo et al., “Targeted biopsy validation of peripheral zone prostate cancer characterization with magnetic resonance fingerprinting and diffusion mapping," Investigative Radiology, vol. 54, no. 8, pp. 485-493, 2019. 
[11] T. Kobayashi, M. S. Orendurff, M. L. Singer, F. Gao, and K. B. Foreman, "Contribution of ankle-foot orthosis moment in regulating ankle and knee motions during gait in individuals post-stroke," Clinical Biomechanics, vol. 45, pp. 9-13, 2017.

[12] M. Carrozzo, G. Vicenti, V. Pesce et al., "Beyond the pillars of the ankle: a prospective randomized CT analysis of syndesmosis' injuries in Weber B and C type fractures," Injury, vol. 49, no. 3, pp. S54-S60, 2018.

[13] K. Yamauchi, A. Yoshiko, S. Suzuki et al., "Muscle atrophy and recovery of individual thigh muscles as measured by magnetic resonance imaging scan during treatment with cast for ankle or foot fracture," Journal of Orthopaedic Surgery (Hong Kong), vol. 25, no. 3, 2017.

[14] J. H. You, I. H. Kim, J. Hwang, H. S. Lee, and E. H. Park, "Fracture of ankle: MRI using opposed-phase imaging obtained from turbo spin echo modified Dixon image shows improved sensitivity," The British Journal of Radiology, vol. 91, no. 1088, Article ID 20170779, 2018.

[15] S. Persaud, M. J. Hentges, and A. R. Catanzariti, "Occurrence of lateral ankle ligament disease with stage 2 to 3 adult-acquired flatfoot deformity confirmed via magnetic resonance imaging: a retrospective study," The Journal of Foot and Ankle Surgery, vol. 58, no. 2, pp. 243-247, 2019.

[16] T. O. Clanton, C. P. Ho, B. T. Williams et al., "Magnetic resonance imaging characterization of individual ankle syndesmosis structures in asymptomatic and surgically treated cohorts," Knee Surgery, Sports Traumatology, Arthroscopy, vol. 24, no. 7, pp. 2089-2102, 2016.

[17] C. Eechaute, L. Leemans, M. De Mesmaeker et al., "The predictive value of the multiple hop test for first-time noncontact lateral ankle sprains," Journal of Sports Sciences, vol. 38, no. 1, pp. 86-93, 2020.

[18] C. Razzano, R. Izzo, R. Savastano, C. Colantuoni, and S. Carbone, "Noninvasive interactive neurostimulation therapy for the treatment of low-grade lateral ankle sprain in the professional contact sport athlete improves the short-term recovery and return to sport: a randomized controlled trial," The Journal of Foot and Ankle Surgery, vol. 58, no. 3, pp. 441-446, 2019. 